Research Article

\title{
Optimized Extraction Method for Kleeb Bua Daeng Formula with the Aid of the Experimental Design
}

\author{
Nittaya Ngamkhae, ${ }^{1}$ Orawan Monthakantirat, ${ }^{2}$ Yaowared Chulikhit, ${ }^{2}$ \\ Chantana Boonyarat, ${ }^{2}$ Charinya Khamphukdee, ${ }^{2}$ Juthamart Maneenat $\mathbb{D},{ }^{1}$ \\ Pakakrong Kwankhao, ${ }^{3}$ Supaporn Pitiporn, ${ }^{3}$ and Supawadee Daodee $\mathbb{D}^{2}$ \\ ${ }^{1}$ Graduate School of Pharmaceutical Sciences, Khon Kaen University, Khon Kaen 40002, Thailand \\ ${ }^{2}$ Division of Pharmaceutical Chemistry, Faculty of Pharmaceutical Sciences, Khon Kaen University, Khon Kaen 40002, Thailand \\ ${ }^{3}$ Department of Pharmacy, Chao Phya Abhaibhubejhr Hospital, Ministry of Public Health, Tha Ngam, \\ Prachinburi 25000, Thailand
}

Correspondence should be addressed to Supawadee Daodee; csupawad@kku.ac.th

Received 28 May 2021; Accepted 16 August 2021; Published 25 August 2021

Academic Editor: Lucica Barbes

Copyright (c) 2021 Nittaya Ngamkhae et al. This is an open access article distributed under the Creative Commons Attribution License, which permits unrestricted use, distribution, and reproduction in any medium, provided the original work is properly cited.

\begin{abstract}
Kleeb Bua Daeng formula is one of the popular remedies sold in Chao Phya Abhaibhubejhr Hospital, Thailand. This formula contains Piper nigrum L., Nelumbo nucifera Gaertn., and Centella asiatica L. as active components. Owing to getting the highest content of its phytochemical compounds, the conditions of solvent extraction for this formula were optimized. The type of solvent, number of extraction times, and ratio between the material and solvent were varied in this study using the Box-Behnken design. The important phytochemical constituents (total phenolics, flavonoids, carotenoids, and anthocyanins) were also determined. From the result of this study, it was found that the highest content of each total active compound was obtained from different conditions such as the optimal extraction condition of phenolic content was obtained using methanol as solvent, one time of extraction, and the ratio of powder and solvent was $1: 6$. Thus, the variation of solvent extraction condition could affect the phytochemical content. Further studies about the herbal formula involving the extraction process should concern the variation of extraction conditions to get the highest content of the active compound.
\end{abstract}

\section{Introduction}

Kleeb Bua Daeng formula (KBD) is one of the Thai traditional herbal formula products by Chao Phya Abhaibhubejhr Hospital, Prachinburi Province, Thailand. Three main components are Piper nigrum L. fruit, Nelumbo nucifera Gaertn. petal, and Centella asiatica L. leaf. This product was claimed to help insomnia improvement and support memory. Black pepper (Piper nigrum L.) is a medicinal plant in family Piperaceae. Pharmacological activities of Piper nigrum fruit were reviewed such as anticancer, antidepressant, anti-inflammatory, analgesic, antioxidant, and antimicrobial [1-5]. The bioactive compounds such as polyphenols, piperine, and essential oils were also reported $[1,6]$. Nelumbo nucifera Gaertn. (NN) is an aquatic plant in family Nelumbonaceae. Various parts of this plant has been reported and used for the anti-inflammatory, antioxidant, neuroprotective, anticancer, and antibacterial activities [7-12]. The bioactive compounds found in the petal part were flavonoids (e.g., quercetin) and anthocyanins (e.g., cyanidin-3-O-glucoside) [13-17]. Centella asiatica L. (CA) also known as Kotu Gola is medicinal plant in family Apiaceae commonly used in the treatment of wound healing and as an anti-inflammatory, antioxidant, and neuroprotective agent [18-21]. The active constituents found in the aerial part are asiaticoside, polyphenols, flavonoids, and carotenoids [22, 23]. Many techniques can be used for the extraction of the active component in herbal remedy or herbal plant such as solvent extraction, ultrasound-assisted extraction, microwave-assisted extraction, and supercritical 
fluid extraction. One of the important basic and versatile techniques used for a long time is the solvent extraction method. The principle of solvent extraction is to separate compounds based on their relative solubility in two immiscible solvents, usually one polar and one nonpolar. The advantages of this technique are that it is considered widely, simple, low cost, and requires uncomplicated utensil and equipment [24]. Many factors or variables for solvent extraction should be optimized in which the extraction is an important process for herbal product development to obtain high content of active phytochemical compounds. KBD formula, one of the popular products by Chao Phya Abhaibhubejhr Hospital, is the interesting product in the list of product development which needs to find a new formula from the extract.

Experimental design is one of the methods to study the various factors' or parameters' effect on the results of the study. The response surface methodology (RSM) is one of the effective statistical analysis techniques which comprises several statistical designs such as the Box-Behnken Design (BBD), Central Composite Design (CCD), Optimal Design, and other statistical procedures. Among these designs, the BBD-based statistical modeling represents a simplified design to cover three levels of experimental factors with a smaller number of experiments [25]. BBD was developed in 1960 by Box and Behnken. This design can be applied to use in the issues having three or more factors, and each has three levels [26]. BBD is easy to perform experiments and interpret in comparison to other models. RSM is an effective statistical tool optimizing complex processes and widely used for optimizing the extraction [27]. This method could evaluate the interactions among various factors, as well as simultaneously estimating the effects of several process variables and their interactions with response variables [28]. Optimization by the response surface design is one of the chemometric approaches which endorse the optimal condition of the method. From the study of Dhawan in 2018 [29], a definitive screening design and response surface methodology were used in order to maximize the extraction yield of an Indian coal by optimization of acid pretreatment conditions [29].

Thus, in this study, optimization of the variable factor for solvent extraction of KBD formula using the response surface methodology (RSM) with Box-Behnken design (BBD), which is an effective statistical method to find optimum processing parameters, was studied in order to maximize the extraction yield and total active contents. The best extraction condition in this study could give the highest active compound which could be used for the product development in the future.

\section{Materials and Methods}

2.1. Materials and Equipment. Thai Traditional herbal formula used in this study is the KBD Formula, which contains 3 herbs: Centella asiatica L. leaf (voucher specimen ABH17), Nelumbo nucifera Gaertn. petal (voucher specimen ABH15), and Piper nigrum L. fruit (voucher specimen ABH18), and their voucher specimens were deposited at the Chao Phya
Abhaibhubejhr Hospital Foundation Under the Royal Patronage of H.R.H. Princess Bejraratanarajsuda, Prachinburi Province, Thailand. Folin-Ciocalteu phenol reagent, $\beta$-carotene, sodium acetate, and quercetin were purchased from Sigma-Aldrich (Germany), ethanol, methanol, and ethyl acetate were purchased from VWR Chemicals BDH (France), and gallic acid, hexane, and hydrochloric acid were purchased from Merck (Germany). Sodium carbonate (Loba chemie, India), aluminum chloride (Ajax Finechem, Australia), potassium chloride (QRëC, New Zealand), acetone (RCI Labscan, Thailand), and ultrapure water were also used in this study. A rotary vacuum evaporator (Buchi, Germany), a freeze dryer (Labconco, USA), and an incubator microplate reader (PerkinElmer, Inc, USA) were used.

\subsection{Experimental Design. Seventeen laboratory experiments} at three levels $(-1,0,+1)$ and three factors of BBD with RSM were used to evaluate the optimal conditions of the extraction process. The experimental design of this study was performed using Design-Expert software (Free Trial Version 12, Stat-Ease Inc., Minneapolis, USA). All experiments were performed in triplicate. The factors and their levels are shown in Table 1 . Parameters or factors for this study were the type of solvent, number of extraction times, and material-to-solvent ratio.

The type of solvent is one of the most important parameters for extraction study as an active compound could be dissolved in different types of solvent depending on its solubility which ultimately affects the percentage of extraction yield and total active content. Different types of solvent mean different polarities which effects the extraction yield and the active compound content dissolved in each solvent. The number of extraction times was the other parameter which was concerned to improve the efficiency of extraction as the aspect of countercurrent extraction. Another important parameter that was optimized was the material-to-solvent ratio. This parameter was kept from $1: 3$ to $1: 9$. Even though excessive solvent seems to get the high extraction yield, it becomes more economical. Based on the responses of the screening design, three-level continuous factors were developed.

The experimental design was determined using DesignExpert software (Free Trial Version 12, Stat-Ease Inc., USA).

2.3. Extraction Process. Seventeen experimental sets were used to extract KBD powder material. The KBD powder $(5 \mathrm{~g})$ was taken in a $100 \mathrm{ml}$ round-bottom flask and extracted along with each set of variable factors. After that, the extract solutions were filtered, evaporated with a rotary evaporator at $40^{\circ} \mathrm{C}$, and freeze-dried for $24 \mathrm{hr}$. Percentage yield of crude extract was calculated for each set of experiment. The extracts were kept at $-20^{\circ} \mathrm{C}$ prior to analysis.

2.4. Determination of Percentage Extraction Yield. The percentage extraction yield of KBD Formula was calculated by the following equation: 
TABLE 1: Box-Behnken design for the optimization of extraction conditions.

\begin{tabular}{|c|c|c|c|c|}
\hline \multirow{2}{*}{ Factor } & \multirow{2}{*}{ Symbol } & \multicolumn{3}{|c|}{ Levels } \\
\hline & & -1 & 0 & 1 \\
\hline Type of solvent & $A$ & Ethyl acetate & Ethanol & Methanol \\
\hline Number of extraction times (times) & $B$ & 1 & 2 & 4 \\
\hline Material-to-solvent ratio $(\mathrm{g} / \mathrm{ml})$ & $C$ & $1: 3$ & $1: 6$ & $1: 9$ \\
\hline
\end{tabular}

$$
\text { Extraction yield }(\%)=\frac{W_{c}}{W_{s}} \times 100,
$$

where $W_{c}$ is the mass of $\mathrm{KBD}$ crude extract and $W_{s}$ is the mass of the sample.

2.5. Phytochemical Analysis. Total phenolic content in KBD formula was determined using the Folin-Ciocalteu method as described by Singleton and Rossi in 1965 [30]. Briefly, $20 \mu \mathrm{l}$ of the extract solution (1 mg of extract dissolved in $1,000 \mu \mathrm{l}$ of ethanol) was added into $100 \mu \mathrm{l}$ of Folin-Ciocalteu reagent (10\%) and $80 \mu \mathrm{l}$ of sodium carbonate (7\%). After 30 minutes of incubation in the dark, the absorbance was measured at $760 \mathrm{~nm}$ by using a microplate reader. Total phenolic content was expressed as milligram gallic acid equivalents per gram of extract (mg GAE/g extract).

Total flavonoid content in the KBD Formula was determined using the aluminum chloride colorimetric method [31]. Briefly, $20 \mu \mathrm{l}$ of the extract solution was added to $15 \mu \mathrm{l}$ of aluminum chloride, $20 \mu \mathrm{l}$ of sodium acetate (10\%), and $145 \mu \mathrm{l}$ distilled water. After 15 minutes of incubation in the dark, the absorbance was measured at $430 \mathrm{~nm}$ by using a microplate reader. Total flavonoid content was expressed as milligram quercetin equivalents per gram of extract (mg QE/ g extract).

Total carotenoid content was also determined in the KBD Formula. Briefly, $100 \mu \mathrm{l}$ of the extract solution (pretreatment by extraction with ethanol, acetone, and hexane) was added into a 96-well plate, and the absorbance was measured at $450 \mathrm{~nm}$ by using a microplate reader [32]. Total carotenoid content was expressed as milligram $\beta$-carotene equivalents per gram of extract ( $\mathrm{mg} \beta$-carotene/g extract).

Total anthocyanin content in the KBD formula was determined using the $\mathrm{pH}$ differential method as described and modified from the work of Lee et al. [33]. Briefly, $20 \mu$ of extracts was diluted with $100 \mu \mathrm{l}$ of $0.025 \mathrm{M}$ potassium chloride solution at $\mathrm{pH} 1$. Similarly, the extracts were diluted with $100 \mu \mathrm{l}$ of $0.4 \mathrm{M}$ sodium acetate at $\mathrm{pH}$ 4.5. The absorbance of these solutions was measured at 535 and $700 \mathrm{~nm}$ by using a microplate reader. Total anthocyanin content was expressed as milligram cyanidin-3-glucoside equivalents per gram of extract (mg C3G/g extract).

\section{Results and Discussion}

3.1. Fitting the Model. BBD with the response surface method (RSM) was used to optimize solvent extraction factors. The results from analysis of variance (ANOVA) are shown in Table 2 . The analysis of variance method was used to analyze the results in order to obtain an adequate elution model. The coefficient of determination $\left(R^{2}\right)$ showed a good relationship for all variable factors (Figure 1 ). This figure also represented the observed response values compared with that of the predicted values depicting a good fit. The model showed high F-value which implied this model was significant and $P$ value was less than 0.05 indicating the model term was also significant. $R^{2}$ and adjusted $R^{2}$ values were higher than 0.75 for extraction yield, TPC, TFC, TCC, and TAC, respectively. Generally, higher $R^{2}$ could better predict the results from the equation and $R^{2}$ should have at least $0.75[34,35]$. The effect from all variable factors (type of solvent, number of extraction times, and ratio between material and solvent) on the response results was described as a quadratic model in which there was the corelationship between each factor. Comparative results between the predicted value and experimental value which verified the good strength of model fitting are shown in Table 3.

\subsection{Effect of Extraction Factors on Percentage Extraction Yield.} Before using the data to integrate for the relationship of factors, we had to conduct model adequate checking by three categories which were an independence test, normality test, and variance stability test, as shown in Figure 2. From the figure, it can be seen that the experimental data were accurate and suitable to be used for the analysis of coefficient of determination and the analysis of variance.

The appropriate quadratic model for extraction yield showed a significant linear and quadratic effect shown by the following equation:

$$
\begin{aligned}
Y= & +6.36+4.65 A+1.38 B+1.72 C+1.53 A B+1.49 A C \\
& -0.33 B C+2.16 A^{2}-0.78 B^{2}-0.56 C^{2}
\end{aligned}
$$

where $Y$ is the percentage extraction yield (\%), $A$ is the type of solvent, $B$ is the number of extraction times, $C$ is the material-to-solvent ratio, $A, B$, and $C$ are linear terms of factors; $A B, A C$, and $B C$ are interaction terms of factors; and $A^{2}, B^{2}$, and $C^{2}$ are quadratic terms of factors. Most of the terms had a significant effect on response with $P$ value less than 0.05 (percentage extraction yield) except $B C$ and $C^{2}$. From the high values of $R^{2}$ and adjusted $R^{2}$ (Table 2), it could be concluded that the suggested models fit the obtained experimental data adequately. Large $F$-value of this model implied the model was significant.

The result showed the extraction yield was found between $2.15-16.14 \%$. The response surface method was used to explain the relationship between these three variable factors. The interaction effect between the type of solvent and number of extraction times $(A B)$ and the interaction 
TABLE 2: The results from response surface model of percentage extraction yield, TPC, TFC, TCC, and TAC by analysis of variance (ANOVA).

\begin{tabular}{|c|c|c|c|c|c|}
\hline Response & Model & $F$-value & $P$ value & $R^{2}$ & Adjusted $R^{2}$ \\
\hline Percentage extraction yield & Quadratic & 92.87 & $<0.0001^{*}$ & 0.9917 & 0.9810 \\
\hline Total phenolic content & Quadratic & 30.14 & $<0.0001^{*}$ & 0.9748 & 0.9425 \\
\hline Total flavonoid content & Quadratic & 20.18 & $0.0003^{*}$ & 0.9629 & 0.9152 \\
\hline Total carotenoid content & Quadratic & 6.22 & $0.0124^{*}$ & 0.8889 & 0.7460 \\
\hline Total anthocyanin content & Quadratic & 144.87 & $<0.0001^{*}$ & 0.9947 & 0.9878 \\
\hline
\end{tabular}

Level of significance: * significant at $P<0.05$.

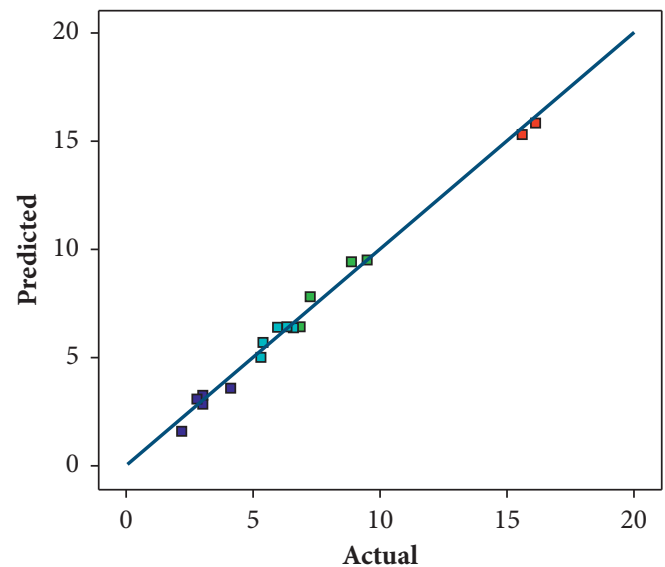

(a)

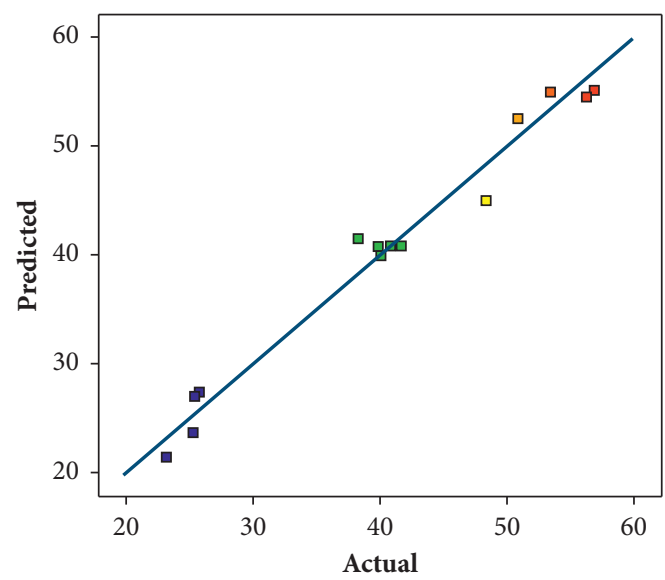

(c)

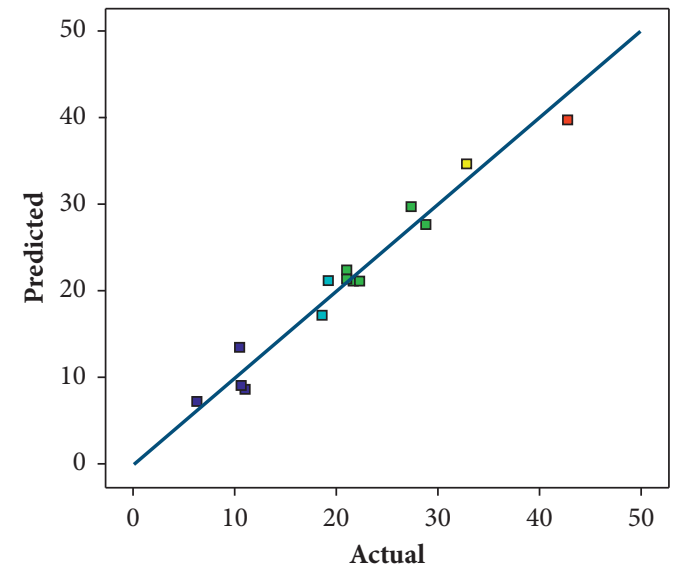

(b)

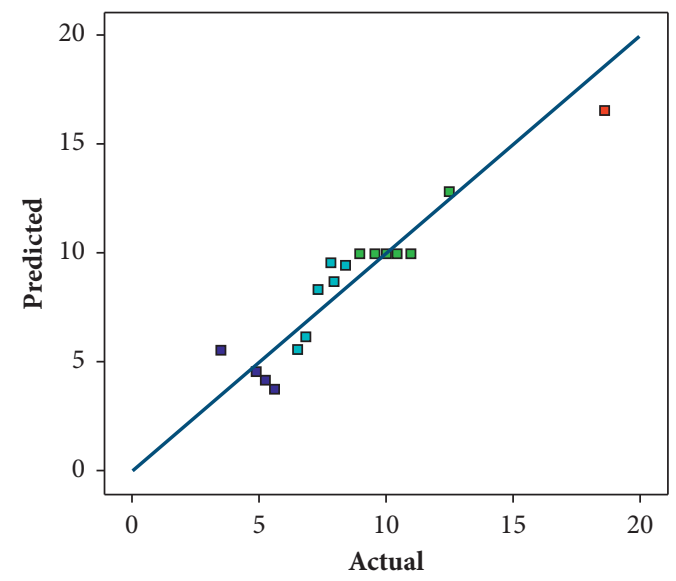

(d)

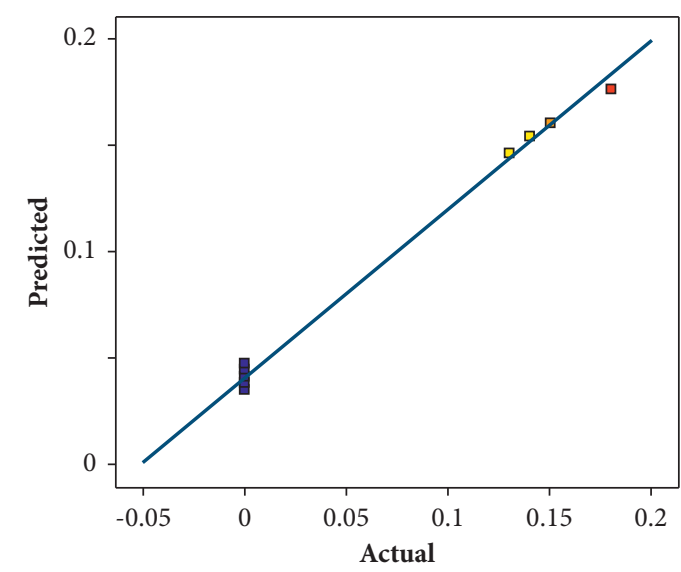

(e)

FIGURE 1: Plot of the relationship between the predicted value from the model and the observed or experimental value from the experiment in each response $(\mathrm{I}=$ percentage extraction yield, $\mathrm{II}=$ total flavonoid content, $\mathrm{III}=$ total phenolic content, $\mathrm{IV}=$ total carotenoid content, and $\mathrm{V}=$ total anthocyanin content). 


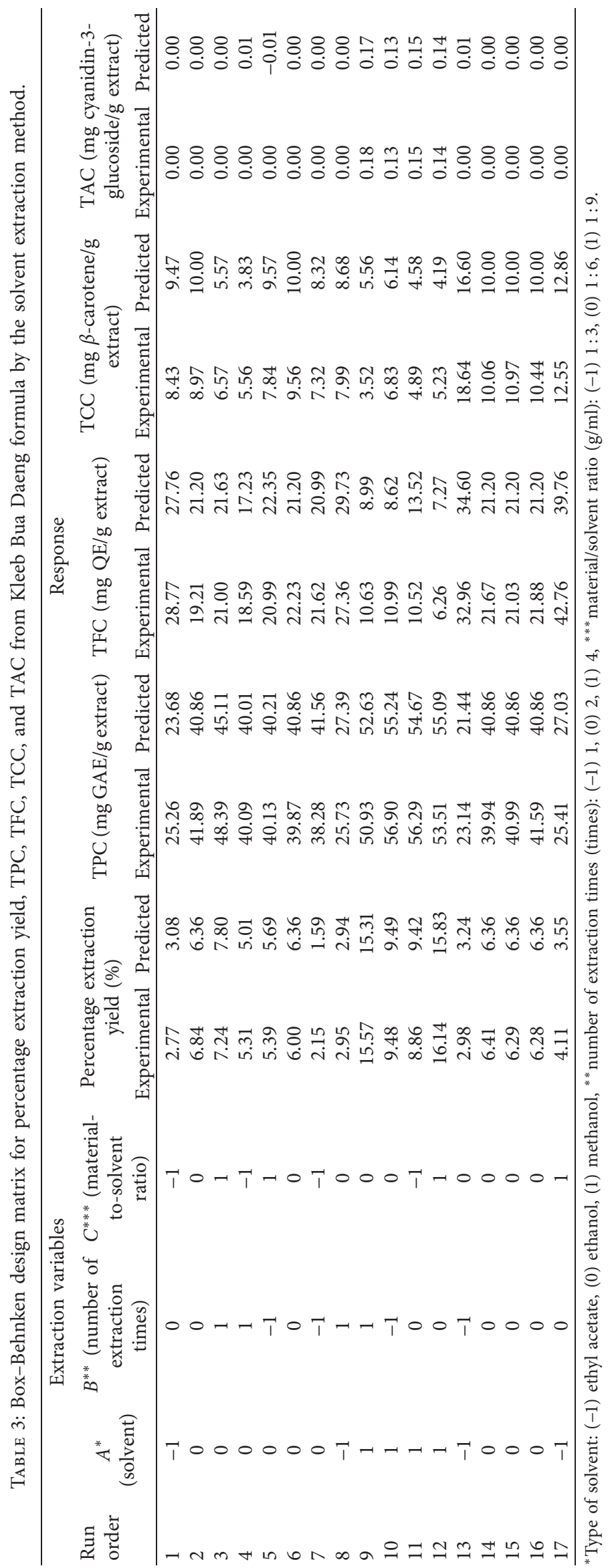



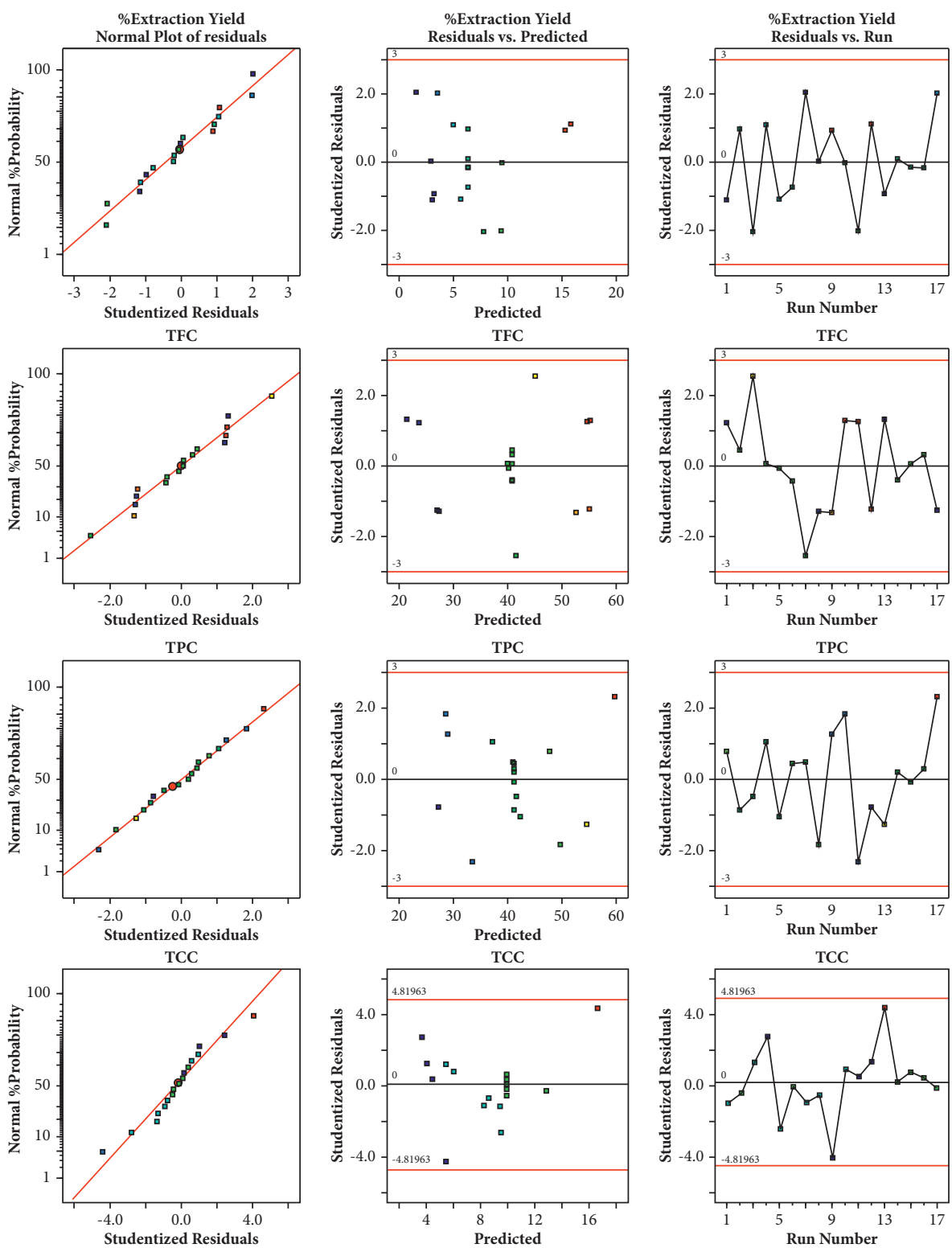

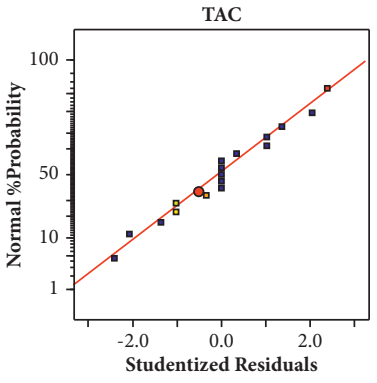

(a)

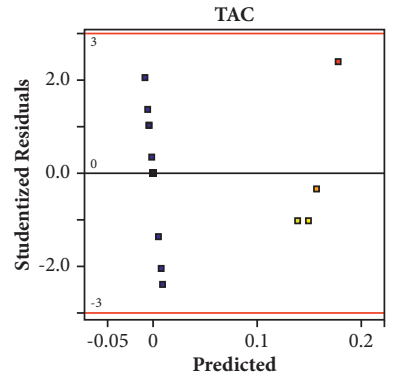

(b)

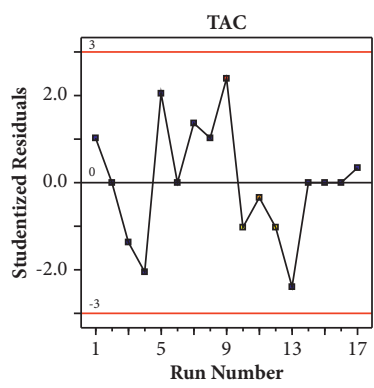

(c)

FIGURE 2: Diagnostic plot for model adequate checking of percentage extraction yield (\%), TPC, TFC, TCC, and TAC: (a) normal \% probability against internally studentized residuals, (b) internally studentized residuals against predicted, and (c) internally studentized residuals against run number.

effect between type of solvent and material-to-solvent ratio $(A C)$ on the extraction yield were demonstrated and showed significance at $P<0.05$ but not for the interaction effect between the number of extraction times and material-to-solvent ratio $(B C)$. Thus, the response (percentage extraction yield) was not affected by the interaction between the number of extraction times and material-tosolvent ratio. 
The results of this study also showed the highest the extraction yield $(16.14 \%)$ of the $12^{\text {th }}$ run on the condition (methanol, 2 times the number of extraction times, and 1 : $9 \mathrm{~g} / \mathrm{ml}$ of material-to-solvent ratio) presented in Table 3. The type of solvent showed the most enhancing effect to the extraction yield. The extraction yield increased with the increase in the polarity of solvent used. The solubility of some substances in methanol might be higher than in ethanol and ethyl acetate [36]. Increasing material-to-solvent ratio gave higher diffusion coefficient [37]. The number of extraction times affected the efficiency of extraction which might be explained as the principle of countercurrent extraction [38]. Even though the highest extraction yield was performed by methanol as a solvent, the better use for preparing the extract as the herbal product composition should provide the lowest toxicity. Thus, ethanol is the most effective and appropriate solvent for using in this herbal product formulation even though it may not show the highest efficiency for some phytochemical compounds such as anthocyanins.

\subsection{Effect of Extraction Factors on Total Phenolic Content} (TPC). The quadratic model for total phenolic content showed a significant linear and quadratic effect. The secondorder polynomial equation (3) is

$$
\begin{aligned}
Y= & +40.86+14.76 A+0.84 B+0.94 C-2.14 A B-0.73 A C \\
& +1.61 B C-1.64 A^{2}-0.038 B^{2}+0.90 C^{2},
\end{aligned}
$$

where $Y$ is the total phenolic content and the other terms ( $A$, $B, C, A B, A C, B C, A^{2}, B^{2}$, and $C^{2}$ ) were also described above. The results of the statistical significance of every regression coefficient are presented in Table 2. The model $P$ value of $<0.05$ for total phenolic content was revealed. In a similar manner, model adequate checking of total phenolic content was also performed as shown in Figures 2(a)-2(c).

Total phenolic content was found between 23.14-56.90 mg GAE/g extract. The results from the response surface method demonstrated no interaction effect between the type of solvent and number of extraction times $(A B)$, type of solvent and material-to-solvent ratio $(A C)$, and number of extraction times and material-to-solvent ratio (BC) on the total phenolic content which were not significant at $P>0.05$. Thus, these results indicated no interaction effect between these factors to the response (total phenolic content).

The results of this study showed the highest total phenolic content $\left(56.90 \mathrm{mg} \mathrm{GAE} / \mathrm{g}\right.$ extract) at the $10^{\text {th }}$ run on the condition (methanol, 1 time the number of extraction times, and $1: 6 \mathrm{~g} / \mathrm{ml}$ of material-to-solvent ratio) presented in Table 3. The type of solvent enhanced and performed the most effect to the amount total phenolic content. The total phenolic content was higher in polar solvents. In this study, the total phenolic content depends on the type of solvent used, polarity index, and the solubility. The solubility of polyphenols depends on the hydroxyl groups, molecular size, and length of the hydrocarbon [39], as increasing material-to-solvent ratio gave higher diffusion coefficient [37]. Moreover, the number of extraction times was increasing affecting the total phenolic content.

\subsection{Effect of Extraction Factors on Total Flavonoid Content} (TFC). The quadratic model for total flavonoids content showed significant linear and quadratic effect. The secondorder polynomial equation (4) is

$$
\begin{aligned}
Y= & +21.20-11.68 A-1.12 B+1.44 C+1.31 A B-4.56 A C \\
& +0.76 B C+0.40 A^{2}-1.12 B^{2}+0.47 C^{2},
\end{aligned}
$$

where $Y$ is the total flavonoid content and the other terms were described above. The results of the statistical significance of every regression coefficient are presented in Table 2 . The model showed $P$ value of $<0.05$ for total flavonoids content which revealed the influencing effect of all variable factors on total flavonoid content. Figures 2(a)-2(c) show the data normality and model adequate checking.

Total flavonoid content was found between 6.26-42.76 mg QE/g extract and demonstrated the interaction effect between each factor. Only a significant interaction effect between the type of solvent and material-tosolvent ratio $(A C)$ was observed with $P$ value $<0.05$ while the others $(B C$ and $A B$ ) were not. Moreover, the type of solvent showed the most effective factor to the flavonoid content as shown by the highest coefficient of this factor from the fitting equation (equation (4)).

The results of this study showed the highest the total flavonoid content $(42.76 \mathrm{mg} \mathrm{QE} / \mathrm{g}$ extract) of the 17 th run on the condition (ethyl acetate, 2 times the number of extraction times, and $1: 9 \mathrm{~g} / \mathrm{ml}$ of material-to-solvent ratio) presented in Table 3 . The type of solvent enhances the amount of total flavonoid content which means the medium polarity of solvent is likely to dissolve the flavonoid compound from this sample. Polar solvents are used to obtain flavonoid glycosides, whereas nonpolar solvents extracted mostly their aglycones. Flavonoids have a diversity of chemical structures constituted of 15 carbon atoms in their basic skeletons with a C6-C3-C6. The chemical structure diversity of flavonoids is particularly obtained from glycosylation, methoxylation, prenylation, and hydroxylation that usually took place with some specific positions [40]. This result showed the flavonoid content in this sample could be extracted by ethyl acetate, ethanol, and methanol because of the mix type of flavonoid in our sample, and the results would be different for the other types of herbal sample. Among the examples of solvents for the extraction of herbal tea from Viscum album L., ethanol, methanol, and acetone were among the best solvents for extracting flavonoids from this tea. As increasing material-to-solvent ratio gave higher diffusion coefficient [37], higher volume of solvent can better improve the penetration into the material particle. The number of extraction times was increasing affecting the total flavonoid content. 
3.5. Effect of Extraction Factors on Total Carotenoid Content (TCC). The quadratic model for total carotenoid content showed a significant linear and quadratic effect. The secondorder polynomial equation (5) is

$$
\begin{aligned}
Y= & +10.00-3.39 A-2.12 B+0.75 C+1.83 A B-0.95 A C \\
& +0.12 B C+0.099 A^{2}-0.85 B^{2}-2.32 C^{2}
\end{aligned}
$$

where $Y$ is the total carotenoid content and the other terms were described above. The results of statistical significance of every regression coefficient are presented in Table 2. The model showed a $P$ value of 0.0124 for this response which showed a significant effect. Normal percentage of probability against internally studentized residuals, internally studentized residuals against predicted, and internally studentized residuals against run number of total carotenoid content was used to check the distribution of residuals, as shown in Figures 2(a)-2(c).

Total carotenoid content was found between 3.52-18.64 mg $\beta$-carotene/g extract. No interaction effect between these factors $(A B, A C$, and $B C)$ was found. However, the type of solvent and number of extraction times $(A B)$ showed the highest effect as performed by the highest coefficient of this term in equation (5).

The results of this study showed the highest total carotenoid content $\left(18.64 \mathrm{mg} \beta\right.$-carotene $/ \mathrm{g}$ extract) of the $13^{\text {th }}$ run on the condition (ethyl acetate, 1 times the number of extraction times, and $1: 6 \mathrm{~g} / \mathrm{ml}$ of material-to-solvent ratio) presented in Table 3 . The type of solvent enhances the amount of total carotenoid content. The total carotenoid content was higher in ethyl acetate. In general, the nonpolar solvent seems to enhance the solubility of the nonpolar carotenoids (beta-carotene) [41]. Increasing material-tosolvent ratio gave higher diffusion coefficient [37], hence increasing the carotenoid content. The number of extraction times was also increasing the total carotenoid content.

3.6. Effect of Extraction Factors on Total Anthocyanin Content (TAC). The quadratic model for total anthocyanin content showed a significant linear and quadratic effect. The secondorder polynomial equation (6) is

$$
\begin{aligned}
Y= & 0.075 A+0.0063 B-0.0012 C+0.012 A B-0.0025 A C \\
& +0.0 .075 A^{2}+0.0025 B^{2}-0.0025 C^{2},
\end{aligned}
$$

where $Y$ is the total anthocyanin content and the other factors were the terms as described above. The results of the statistical significance of every regression coefficient are presented in Table 2. In the same manner as the other factors, the model showed a $P$ value of $<0.0001$ for total anthocyanin content which expressed the effect of all factors on anthocyanin content. The normality of data was also checked as shown in Figures 2(a)-2(c).

Total anthocyanin content was found between 0.00-0.18 mg cyanidin-3-glucoside/g extract) and demonstrated the interaction effect between the type of solvent and number of extraction times $(A B)$ which was significant at $P<0.05$ which revealed the interaction effect between these two factors on response.

The results of this study showed the highest total anthocyanin content ( $0.18 \mathrm{mg}$ cyanidin-3-glucoside/g extract) of the $9^{\text {th }}$ run on the condition (methanol, 4 times the number of extraction times, and $1: 6 \mathrm{~g} / \mathrm{ml}$ of material-tosolvent ratio) presented in Table 3 . The type of solvent enhances the amount of total anthocyanin content. The total anthocyanin content was higher in polar solvents. In general, anthocyanins occur naturally as glycosides, so polar solvents are essential for good extraction [42]. Similarly, increasing material-to-solvent ratio and number of extraction times was also increasing the effect on the total anthocyanin content.

\section{Conclusions}

From our study, the optimal extraction for KBD formula was developed. Many responses as percentage yield and total active content were chosen. The selected variable factors (type of solvent, number of extraction times, and ratio of material to solvent) affected to these responses as from RSM analysis results. A screening of factors based on RSM-BBD analysis revealed the optimal conditions which led to the high level of responses. However, the selection of the optimal condition for each active content to product development should depend on the purpose and use of that formulation. Different types of active compound would express different biological activities. Thus, product development should concern the biological effect and justify the extraction condition to fit the purpose. These results gave the important data to develop a new formula KBD with high percentage yield and the selected active contents. Further study about the optimized extraction method such as ultrasound-assisted extraction or supercritical fluid extraction should be performed to get the better yield of active compound and lead to use the newer technique for saving the time of extraction and less organic solvent as the green extraction technique. Moreover, improving the variable factors from the study for the basic solvent extraction technique might be necessary to get the better suitable variable factors such as changing the type of organic solvent (ethyl acetate, methanol, and ethanol) to the ratio of ethanol and water for the extraction.

\section{Data Availability}

The main part of the data is included within the content. Other data can be obtained from the corresponding author upon request.

\section{Conflicts of Interest}

The authors declare no conflicts of interest regarding the publication of this paper.

\section{Acknowledgments}

This work was financially supported by Khon Kaen University, Thailand (I62-00-21-01 and KKURP: F64010098). 


\section{References}

[1] A. Ahmad, A. Husain, M. Mujeeb, S. A. Khan, H. A. A. Alhadrami, and A. Bhandari, "Quantification of total phenol, flavonoid content and pharmacognostical evaluation including HPTLC fingerprinting for the standardization of Piper nigrum L fruits," Asian Pacific Journal of Tropical Biomedicine, vol. 5, no. 2, pp. 101-107, 2015.

[2] Y. Deng, S. Sriwiriyajan, A. Tedasen, P. Hiransai, and P. Graidist, "Anti-cancer effects of Piper nigrum via inducing multiple molecular signaling in vivo and in vitro," Journal of Ethnopharmacology, vol. 188, pp. 87-95, 2016.

[3] L. Hritcu, J. A. Noumedem, O. Cioanca, M. Hancianu, P. Postu, and M. Mihasan, "Anxiolytic and antidepressant profile of the methanolic extract of Piper nigrum fruits in betaamyloid (1-42) rat model of Alzheimer's disease," Behavioral and Brain Functions: BBF, vol. 11, no. 1, p. 13, 2015.

[4] Z. Zarai, E. Boujelbene, N. Ben Salem, Y. Gargouri, and A. Sayari, "Antioxidant and antimicrobial activities of various solvent extracts, piperine and piperic acid from Piper nigrum," Lebensmittel-Wissenschaft und-Technologie-Food Science and Technology, vol. 50, no. 2, pp. 634-641, 2013.

[5] F. Tasleem, I. Azhar, S. N. Ali, S. Perveen, and Z. A. Mahmood, "Analgesic and anti-inflammatory activities of Piper nigrum L," Asian Pacific Journal of Tropical Medicine, vol. 7, no. S1, pp. S461-S468, 2014.

[6] F. Zhu, R. Mojel, and G. Li, "Physicochemical properties of black pepper (Piper nigrum) starch," Carbohydrate Polymers, vol. 181, pp. 986-993, 2018.

[7] S. H. Liu, T. H. Lu, C. C. Su et al., "Lotus leaf (Nelumbo nucifera) and its active constituents prevent inflammatory responses in macrophages via JNK/NF- $\kappa \mathrm{B}$ signaling pathway," American Journal of Chinese Medicine, vol. 42, no. 4, pp. 869-889, 2014.

[8] D.-J. Shin, J. Choe, K.-E. Hwang, C.-J. Kim, and C. Jo, "Antioxidant effects of lotus (Nelumbo nucifera) root and leaf extracts and their application on pork patties as inhibitors of lipid oxidation, alone and in combination," International Journal of Food Properties, vol. 22, no. 1, pp. 383-394, 2019.

[9] E. S. Kim, J. B. Weon, B. R. Yun et al., "Cognitive enhancing and neuroprotective effect of the embryo of the Nelumbo nucifera seed," Evidence-Based Complementary \& Alternative Medicines, vol. 2014, Article ID 869831, 9 pages, 2014.

[10] T. Prabsattroo, J. Wattanathorn, P. Somsapt, and O. Sritragool, "Positive modulation of pink Nelumbo nucifera flowers on memory impairment, brain damage, and biochemical profiles in restraint rats," Oxidative Medicine o Cellular Longivity, vol. 2016, Article ID 5789857, 11 pages, 2016.

[11] X. Zhao, X. Feng, C. Wang, D. Peng, K. Zhu, and J.-L. Song, "Anticancer activity of Nelumbo nucifera stamen extract in human colon cancer HCT-116 cells in vitro," Oncology Letters, vol. 13, no. 3, pp. 1470-1478, 2016.

[12] D. Brindha and D. Arthi, "Antimicrobial activity of white and pink Nelumbo nucifera gaertn flowers," Asian Journal of Pharmaceutical Research and Health Care, vol. 2, pp. 147-155, 2010.

[13] S. Chen, L. Fang, H. Xi et al., "Simultaneous qualitative assessment and quantitative analysis of flavonoids in various tissues of lotus (Nelumbo nucifera) using high performance liquid chromatography coupled with triple quad mass spectrometry," Analytica Chimica Acta, vol. 724, pp. 127-135, 2012.
[14] S. Chen, Y. Xiang, J. Deng, Y. Liu, and S. Li, "Simultaneous analysis of anthocyanin and non-anthocyanin flavonoid in various tissues of different lotus (Nelumbo) cultivars by HPLC-DAD-ESI-MS (n)," PLoS One, vol. 8, no. 4, Article ID e62291, 2013.

[15] J. Deng, S. Chen, X. Yin et al., "Systematic qualitative and quantitative assessment of anthocyanins, flavones and flavonols in the petals of 108 lotus (Nelumbo nucifera) cultivars," Food Chemistry, vol. 139, no. 1-4, pp. 307-312, 2013.

[16] S. S. Li, J. Wu, L. G. Chen et al., "Biogenesis of C-glycosyl flavones and profiling of flavonoid glycosides in Lotus (Nelumbo nucifera)," PLoS One, vol. 9, no. 10, Article ID e108860, 2014.

[17] R.-Z. Yang, X.-L. Wei, F.-F. Gao et al., "Simultaneous analysis of anthocyanins and flavonols in petals of lotus (Nelumbo) cultivars by high-performance liquid chromatography-photodiode array detection/electrospray ionization mass spectrometry," Journal of Chromatography A, vol. 1216, no. 1, pp. 106-112, 2009.

[18] H. A. Azis, M. Taher, A. S. Ahmed et al., "In vitro and In vivo wound healing studies of methanolic fraction of Centella asiatica extract," South African Journal of Botany, vol. 108, pp. 163-174, 2017.

[19] J. H. Park, J. Y. Choi, D. J. Son et al., “Anti-inflammatory effect of titrated extract of Centella asiatica in phthalic anhydrideinduced allergic dermatitis animal model," International Journal of Molecular Sciences, vol. 18, no. 4, pp. 1-14, 2017.

[20] A. Kumar, S. Dogra, and A. Prakash, "Neuroprotective effects of Centella asiatica against intracerebroventricular colchicineinduced cognitive impairment and oxidative stress," International Journal of Alzheimer's Disease, vol. 2009, Article ID 972178, 2009.

[21] F. Pittella, R. Dutra, D. Junior, M. T. Lopes, and N. Barbosa, "Antioxidant and cytotoxic activities of Centella asiatica (L) urb,” International Journal of Molecular Sciences, vol. 10, no. 9, pp. 3713-3721, 2009.

[22] B. Brinkhaus, M. Lindner, D. Schuppan, and E. G. Hahn, "Chemical, pharmacological and clinical profile of the East Asian medical plant Centella aslatica," Phytomedicine, vol. 7, no. 5, pp. 427-448, 2000.

[23] K. D. P. P. Gunathilake, K. K. D. S. Ranaweera, and H. P. V. Rupasinghe, "Response surface optimization for recovery of polyphenols and carotenoids from leaves of Centella asiatica using an ethanol-based solvent system," Food Sciences and Nutrition, vol. 7, no. 2, pp. 528-536, 2019.

[24] N. N. Azwanida, "A review on the extraction methods use in medicinal plants, principle, strength and limitation," $\mathrm{Me}$ dicinal \& Aromatic Plants, vol. 4, no. 3, pp. 196-201, 2015.

[25] Q. Zhao, J. F. Kennedy, X. Wang et al., "Optimization of ultrasonic circulating extraction of polysaccharides from Asparagus officinalis using response surface methodology," International Journal of Biological Macromolecules, vol. 49, no. 2, pp. 181-187, 2011.

[26] G. E. Box and D. W. Behnken, "Some new three level designs for the study of quantitative variables," Technometrics, vol. 2, no. 4, pp. 455-475, 1960.

[27] A. Pandey, T. Belwal, K. C. Sekar, I. D. Bhatt, and R. S. Rawal, "Optimization of ultrasonic-assisted extraction (UAE) of phenolics and antioxidant compounds from rhizomes of Rheum moorcroftianum using response surface methodology (RSM)," Industrial Crops and Products, vol. 119, pp. 218-225, 2018.

[28] J. Prakash Maran, S. Manikandan, K. Thirugnanasambandham, C. Vigna Nivetha, and R. Dinesh, "Box-behnken design based 
statistical modeling for ultrasound-assisted extraction of corn silk polysaccharide," Carbohydrate Polymers, vol. 92, no. 1, pp. 604-611, 2013.

[29] H. Dhawan, S. Upadhyayula, and D. K. Sharma, "Design of experiments to optimize the extraction parameters of a power grade Indian coal," International Journal of Coal Science \& Technology, vol. 5, no. 4, pp. 417-429, 2018.

[30] V. L. Singleton and J. A. Rossi, "Colorimetry of total phenolics with phosphomolybdic-phosphotungstic acid reagents," American Journal of Enology and Viticulture, vol. 16, pp. 144-158, 1965.

[31] C. Chang, M. Yang, H. Wen, and J. Chern, "Estimation of total flavonoid content in propolis by two complementary colorimetric methods," Journal of Food and Drug Analysis, vol. 10, pp. 178-182, 2002.

[32] M. A. Ashraf, M. J. Maah, and I. Yusoff, "Estimation of antioxidant phytochemicals in four different varieties of durian (Durio zibethinus murray) fruit," Middle-East Journal of Scientific Research, vol. 6, pp. 465-471, 2010.

[33] J. Lee, R. W. Durst, and R. E. Wrolstad, "Determination of total monomeric anthocyanin pigment content of fruit juices, beverages, natural colorants, and wines by the $\mathrm{pH}$ differential method: collaborative study," Journal of AOAC International, vol. 88, no. 5, pp. 1269-1278, 2005.

[34] P. D. Haaland, Biotechnology Experimental Design, Marcel Dekker, Inc., Newyork, NY, USA, 1989.

[35] R. Hu, Food Product Design: A Computer-Aided Statistical Approach, Technomic Publishing Co., Ltd, Lancaster, PA, USA, 1999.

[36] Q. D. Do, A. E. Angkawijaya, P. L. Huynh, F. E. Soetaredjo, S. Ismadji, and Y.-H. Ju, "Effect of extraction solvent on total phenol content, total flavonoid content, and antioxidant activity of Limnophila aromatica," Journal of Food and Drug Analysis, vol. 22, no. 3, pp. 296-302, 2014.

[37] T. Belwal, P. Dhyani, I. D. Bhatt, R. S. Rawal, and V. Pande, "Optimization extraction conditions for improving phenolic content and antioxidant activity in Berberis asiatica fruits using response surface methodology (RSM)," Food Chemistry, vol. 207, pp. 115-124, 2016.

[38] S. Hartland, Counter-Current Extraction-An Introduction to the Design and Operation of Counter-current Extractors, Perkamon Press Ltd, London, UK, 1970.

[39] R. Abarca-Vargas, C. F. Peña Malacara, and V. L. Petricevich, "Characterization of chemical compounds with antioxidant and cytotoxic activities in bougainvillea $\mathrm{x}$ buttiana holttum and standl, (Var. rose) extracts," Antioxidants, vol. 5, no. 4, Article ID 5040045, 2016.

[40] M. D. Awouafack, P. Tane, and H. Morita, Isolation and Structure Characterization of Flavonoids, Flavonoids - from Biosynthesis to Human Health, Intech Open, London, UK, 2017.

[41] R. K. Saini and Y.-S. Keum, "Carotenoid extraction methods: a review of recent developments," Food Chemistry, vol. 240, pp. 90-103, 2018.

[42] S. Oancea, M. Stoia, and D. Coman, "Effects of extraction conditions on bioactive anthocyanin content of Vaccinium corymbosum in the perspective of food applications," Procedia Engineering, vol. 42, pp. 489-495, 2012. 